PROCEEDINGS OF THE

AMERICAN MATHEMATICAL SOCIETY

Volume 126, Number 6, June 1998, Pages 1851-1856

S 0002-9939(98)04364-0

\title{
AN ANNULUS DIFFEOMORPHISM WITH NON-DENJOY MINIMAL SETS
}

\author{
MARK TURPIN
}

(Communicated by Mary Rees)

\begin{abstract}
We construct an annulus diffeomorphism with the property that a countably dense set of irrational rotation numbers are represented only by pseudocircles on which the diffeomorphism acts minimally but is not semiconjugate to rigid rotation on the circle. This answers a question of Boyland about whether such behavior is possible only at the maximum or minimum of the rotation set.
\end{abstract}

\section{INTRODUCTION}

We begin with two notions, rotation number and rotation set, central to the study of annulus homeomorphisms. Given an annulus homeomorphism, the rotation number, $\rho$, of a point is the limiting average angular displacement of the point under iteration, if the limit exists. For an introduction to rotation numbers see, among others, [D].

The rotation set, $\rho(f)$, of a homeomorphism $f$ is the set of numbers realized as rotation numbers. For each member of the rotation set we can ask about the smallest closed invariant sets (minimal sets) with this rotation number. In the case of area preserving monotone twist maps the Aubry-Mather theorem provides an answer to this question. Monotone twist maps are those where points closer to the inner circle of the annulus have a smaller (or larger) angular displacement than points closer to the outer circle of the annulus. (For a complete definition see [Bd].) We state here only the part of the theorem concerning irrational rotation numbers.

Theorem (Aubry-Mather). Let $f$ be an area preserving monotone twist map of the annulus. Then for all $\alpha \in \rho(f)$ with $\alpha \notin \mathbb{Q}, f$ has a monotone, minimal set $X$ with $\rho(f \mid X)=\alpha$ and $f \mid X$ is semiconjugate to rigid rotation on the circle.

An example of Handel $[\mathrm{H}]$ shows that the Aubry-Mather theorem does not extend when the monotone twist condition is removed. Handel constructs a $C^{\infty}$ area preserving diffeomorphism of the annulus with an irrational in the rotation set whose only minimal set (for this irrational) is the pseudocircle [B1], [B2], [F]. This minimal set is not monotone, and the diffeomorphism restricted to the pseudocircle is not semiconjugate to rigid rotation on the circle.

Boyland notes that in Handel's construction the irrational rotation number is the maximum of the rotation set and poses Question 3.3 in [Bd].

Received by the editors June 25, 1996 and, in revised form, November 1, 1996.

1991 Mathematics Subject Classification. Primary 58F13.

(C) 1998 American Mathematical Society 
Question (Boyland). Let $f$ be an area preserving homeomorphism of the annulus. For each irrational, $\alpha$, which lies between the rotation numbers of $f$ restricted to the boundary circles, does $f$ have a minimal set $X$ so that $f \mid X$ is semiconjugate to rigid rotation by $\alpha$ on the circle?

We show that Handel's example can be modified so that the irrational $\alpha$, for which the pseudocircle is the only minimal set, is between the boundary rotation numbers. This provides a negative answer to Boyland's question.

Further, we show that this behavior need not be isolated to a single rotation number.

Theorem. There exists a $C^{\infty}$, area preserving diffeomorphism of the annulus with the property that a countably infinite dense set of irrational rotation numbers are represented only by pseudocircles on which the dynamics are minimal but not semiconjugate to rigid rotation on the circle.

\section{THE CONSTRUCTION}

We remain consistent with the notation used in $[\mathrm{H}]$ and omit here some details of the construction found there. Also, we will work with the universal cover of the annulus $[-1,1] \times \mathbb{R} / \mathbb{Z}$, as reflected in the figures.

Handel's example involves a sequence of nested annuli embedded in $\mathbb{R}^{2}$, and an associated sequence of functions. The first annulus $A_{1}$ is divided into $p_{1}$ pieces, and $f_{1}$ is defined as the rotation which permutes these pieces: $f_{1}(x, y)=\left(x+\frac{1}{p_{1}}, y\right)$. (See Figure 1.)

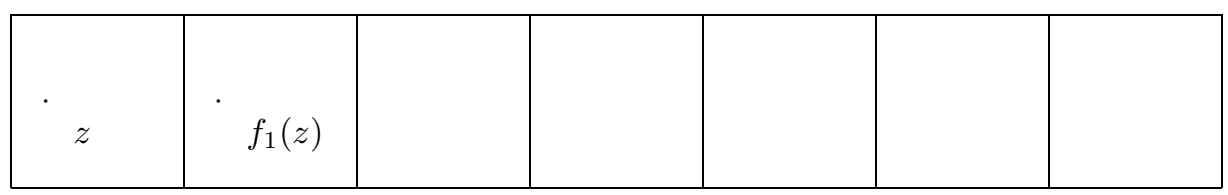

FiguRE 1. $p_{1}=7$

Next we embed a circle $C_{2}$ in $A_{1}$ in a homotopically nontrivial way. (See Figure 2.) Each embedding is done "crookedly" as defined in [B1]. This crookedness, at this and future stages of the construction, is responsible for the "pathological" nature of the intersection of the annuli, the pseudocircle. We also require that $C_{2}$ be invariant under $f_{1}$. The annulus $A_{2}$ is created by thickening the circle $C_{2}$.

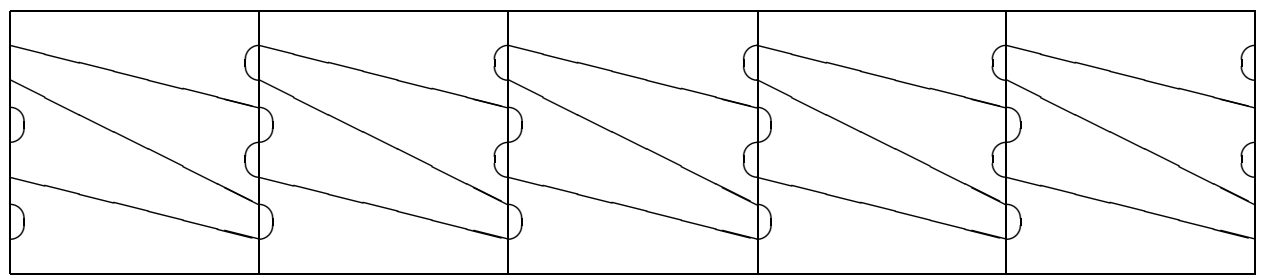

Figure 2. A crooked embedding of $C_{n}$ in $A_{n-1}$

Then $A_{2}$ is divided into $p_{2} \in \mathbb{Z}$ pieces and $f_{2}$ is defined (on $A_{2}$ ) as the composition of $f_{1}$ with the rotation that permutes the $p_{2}$ pieces of $A_{2}$. We modify Handel's 
construction on the definition of $f_{2}$ on the lower part of $A_{1}$ and $A_{2}$. (See Figure 3. Note that in the figures here and to follow, the scale is misrepresented and the crookedness has been suppressed.)

For any circle $C$ we define $U(C)$ as the unbounded component of the complement and $L(A)$ as the bounded component of the complement, or in the universal cover that part of $\mathbb{R} / \mathbb{Z} \times[-1,1]$ which is above or below $C$, respectively. Also, we define $R_{\alpha}(x, y)=(x+\alpha, y)$ as rigid rotation by $\alpha$.

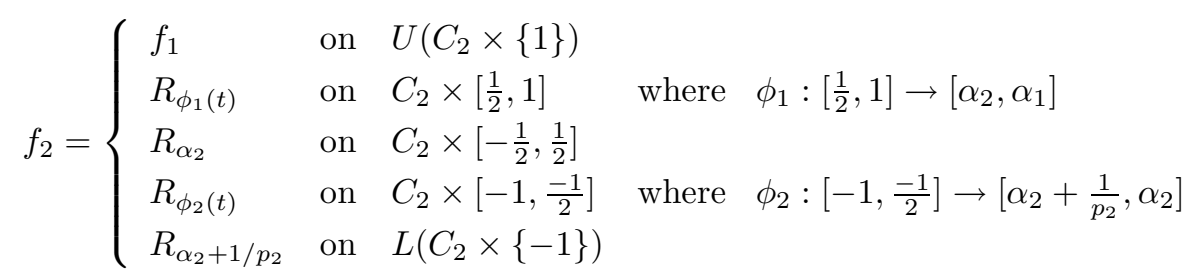

Here we set $\alpha_{2}=\alpha_{1}+\frac{1}{p_{2}}$, and the rotation $R_{\alpha_{2}}$ is a rotation in the annular structure of $A_{2}$.
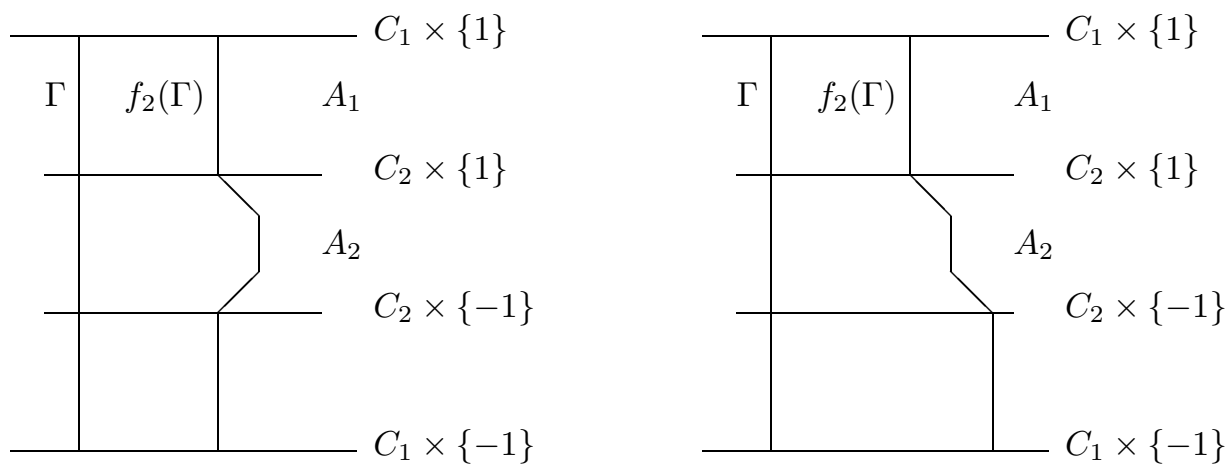

Figure 3. Handel's example(left), Handel's example modified(right)

The construction continues in a like manner. A circle $C_{3}$ is crookedly embedded in $A_{2}$ in a way that leaves it invariant under $f_{2}$. This circle is thickened to form the annulus $A_{3}$ which is then divided into $p_{3}$ pieces. The modified $f_{3}$ is defined (on $\left.A_{3}\right)$ as the composition of $f_{2}$ with the rotation in $A_{3}$ that permutes the $p_{3}$ pieces. Below $A_{3}$ is where the map is modified and differs from Handel's construction.

Continuing, each embedding is accomplished by embedding a circle $C_{n}$ in the annulus $A_{n-1}$ of the previous stage and then thickening $C_{n}$ to form the next annulus $A_{n}$. Each annulus is divided into $p_{n}$ pieces. Then the next annulus is crookedly embedded with the condition that it be invariant under the rotation in the previous annulus. The resulting diffeomorphism is the infinite composition of the $f_{n}$. 
The map $f_{n}$ is defined as follows. (See Figure 4.) Again, $\alpha_{n}=\alpha_{n-1}+\frac{1}{p_{n}}$, each rotation $R$ is in the appropriate annular structure, and the $\phi_{i}$ are diffeomorphisms.

$$
f_{n}=\left\{\begin{array}{lll}
f_{n-1} & \text { on } U\left(\left\{C_{n} \times\{1\}\right\}\right) \\
R_{\phi_{1}(t)} & \text { on } C_{n} \times\left[\frac{1}{2}, 1\right] \quad \text { where } \phi_{1}:\left[\frac{1}{2}, 1\right] \rightarrow\left[\alpha_{n}, \alpha_{n-1}\right] \\
R_{\alpha_{n}} & \text { on } C_{n} \times\left[\frac{-1}{2}, \frac{1}{2}\right] \\
R_{\phi_{2}(t)} & \text { on } C_{n} \times\left[-1, \frac{-1}{2}\right] \quad \text { where } \phi_{2}:\left[-1, \frac{-1}{2}\right] \rightarrow\left[\alpha_{n}+\frac{1}{p_{n}}, \alpha_{n}\right] \\
R_{\alpha_{n}+1 / p_{n}} & \text { on } U\left(C_{n-1} \times\left\{\frac{-1}{2}\right\}\right) \cap L\left(C_{n} \times\{-1\}\right) \\
R_{\phi_{3}(t)} & \text { on } C_{n-1} \times\left[-1, \frac{-1}{2}\right] \quad \text { where } \phi_{3}:[-1,-1 / 2] \\
f_{n-1} & \text { on } L\left(C_{n-1} \times\{-1\}\right) \quad \rightarrow\left[\alpha_{n-1}+\frac{1}{p_{n-1}}, \alpha_{n}+\frac{1}{p_{n}}\right]
\end{array}\right.
$$

We show later that each $f_{n}$ is area preserving and $C^{\infty}$. Given $\epsilon_{n}$ we can choose $p_{n}$ sufficiently large so that $f_{n}$ and $f_{n-1}$ are $\epsilon_{n}$ close in the $C^{n}$ topology. By sending $\epsilon_{n}$ rapidly to 0 we guarantee that the limiting map $f$ restricted to $P$ is $C^{\infty}$.

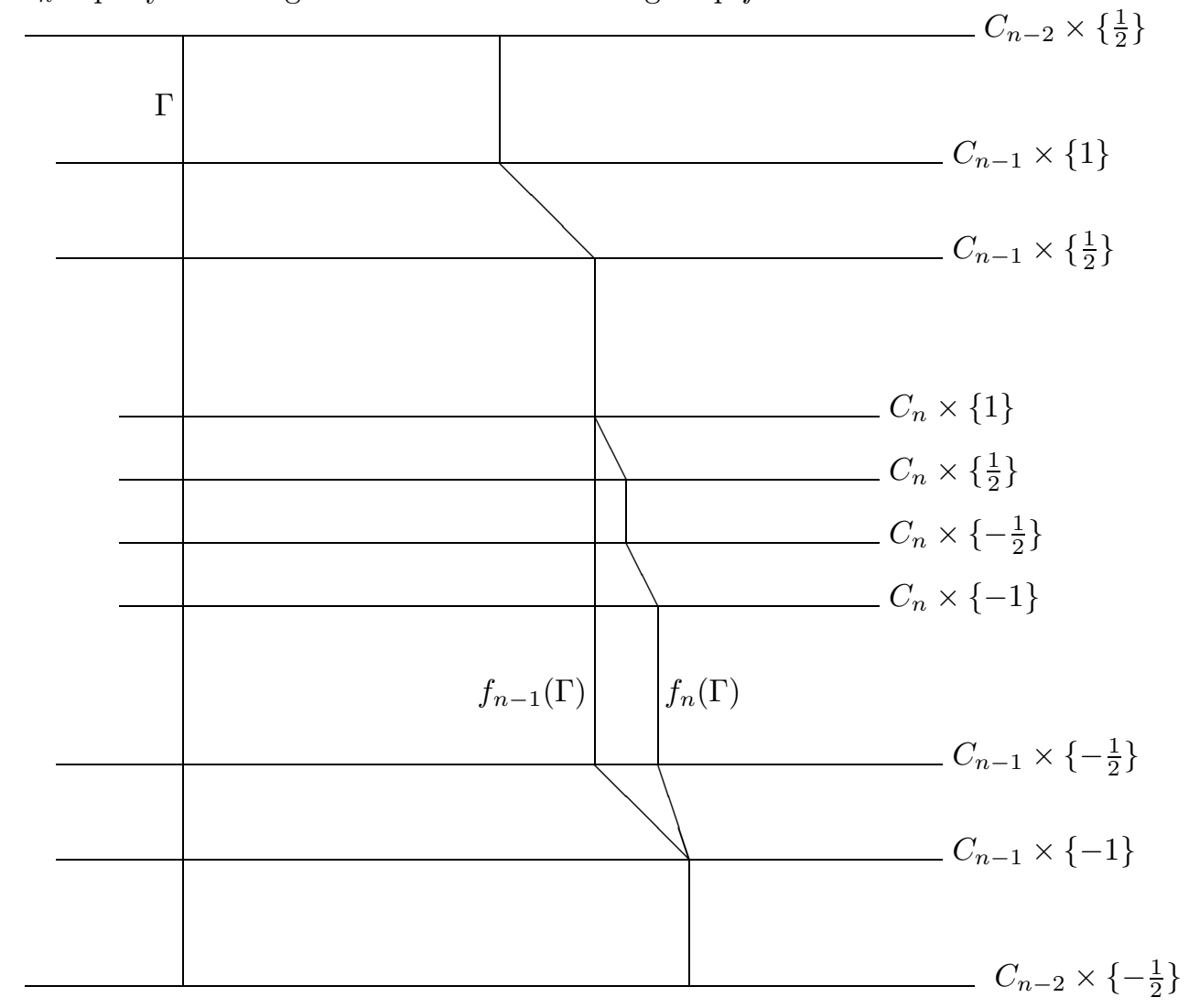

FigurE 4 . Induction step, $f_{n-1} \longrightarrow f_{n}$

We point out that the invariant set $P$ (the intersection of the annuli) and the limiting map $f$ restricted to this invariant set $P$ is exactly as defined in Handel's example. So the topological properties ascribed to $P$, the pseudocircle, remain intact. Also, $P$ remains a minimal set for $f$, and $f \mid P$ has a well defined irrational rotation number even though $f \mid P$ is not semi-conjugate to a rotation of $S^{1}$. What 
remains for us to establish is the greater rotation number below the pseudocircle and the area preservation and differentiability of the limiting map on the entire annulus.

Off the pseudocircle the annulus $A_{1}$ is divided into a sequence of smooth topological annuli and on each of these $f$ is either a finite composition of rotations (on $L\left(C_{n-1} \times\left\{\frac{1}{2}\right\}\right) \cap U\left(C_{n} \times\{1\}\right)$ and $\left.U\left(C_{n-1} \times\left\{-\frac{1}{2}\right\}\right) \cap L\left(C_{n} \times\{-1\}\right)\right)$ or an interpolating map (on $C_{n-1} \times\left[\frac{1}{2}, 1\right]$ and $C_{n-1} \times\left[-1,-\frac{1}{2}\right]$ ). The rotation number of $f$ on these regions is easy to compute.

On $L\left(C_{n-1} \times\left\{\frac{1}{2}\right\}\right) \cap U\left(C_{n} \times\{1\}\right)$ the rotation number of $f$ is $\alpha_{n-1}$ and on $C_{n-1} \times\left[\frac{1}{2}, 1\right]$ interpolates between $\alpha_{n-1}$ and $\alpha_{n-2}$. This is as in Handel's example. On $U\left(C_{n-1} \times\left\{-\frac{1}{2}\right\}\right) \cap L\left(C_{n} \times\{-1\}\right)$ the rotation number of $f$ is $\alpha_{n}+\frac{1}{p_{n}}=\alpha_{n-1}+\frac{2}{p_{n}}$ and on $C_{n-1} \times\left[-1,-\frac{1}{2}\right]$ the rotation number interpolates between $\alpha_{n-1}+\frac{1}{p_{n-1}}=$ $\alpha_{n}+\frac{2}{p_{n-1}}$ and $\alpha_{n}+\frac{1}{p_{n}}$. This differs from Handel's example, and the rapid increase of the sequence $\left\{p_{n}\right\}$ guarantees that $\alpha_{n}+\frac{1}{p_{n}}>\alpha_{n+1}+\frac{1}{p_{n+1}}>\lim _{n \rightarrow \infty} \alpha_{n}=\alpha$ where $\alpha$ is the rotation number of $f \mid P$.

Next we show that the "rotations" $R$ used in the definition of the $f_{n}$ exist as area preserving, $C^{\infty}$ maps conjugate to rigid rotation on the standard annulus. We let $A$ be a smooth topological annulus and $\mu$ be a smooth measure on $A$. We let $\bar{A}=[0,1] \times \mathbb{R} / \mathbb{Z}$ be the standard annulus and $m$ be Lebesgue measure on $\bar{A}$.

From Moser's result $[\mathrm{M}]$ on the equivalence of smooth measures, we have a diffeomorphism $h: A \rightarrow \bar{A}$ with the property that for any $B \subset A$ we have $\mu(B)=$ $m(h(B))$. If $\bar{R}_{\alpha}$ is rigid rotation on $\bar{A}$, then we define $R_{\alpha}=h^{-1} \circ \bar{R}_{\alpha} \circ h$ and this map preserves $\mu$, is smooth, and is conjugate to rigid rotation by $\alpha$.

\section{NUMBER THEORETIC RESTRICTIONS}

The rotation number $\alpha$ of the diffeomorphism restricted to the pseudocircle is the sum of a very rapidly decreasing sequence:

$$
\alpha=\sum_{n=1}^{\infty} \frac{1}{p_{n}} .
$$

In $[\mathrm{T}]$ this rotation number is shown to be very well approximable in the following sense:

$$
\exists C, 1<C<2, \exists\left\{\frac{a_{n}}{b_{n}}\right\}, \frac{a_{n}}{b_{n}} \rightarrow \alpha, \text { and }\left|\alpha-\frac{a_{n}}{b_{n}}\right|<\frac{3}{C^{n}} .
$$

Question 1. Let $f$ be an area preserving homeomorphism of the annulus. For each sufficiently poorly approximable irrational, $\alpha$, in the rotation set, does $f$ have a minimal set $X$ so that $f \mid X$ is semiconjugate to rigid rotation by $\alpha$ on the circle?

\section{Extension to A COUNTABLy Dense subset of the Rotation SET}

We note that in this example there is only one number, the irrational $\alpha$, in the rotation set which is not realized by a "nice" minimal set. However, every other rotation number between the boundary rotation numbers is represented (at least) by a smooth topological circle on which the diffeomorphism is conjugate to rigid rotation. The region between any two such circles with different rotation numbers is an annulus on which the previous construction can be carried out.

Choosing any rational number $\{a / b\}$, and any sufficiently small $\epsilon>0$, we take the region that represents the interval of rotation numbers $[a / b, a / b+\epsilon]$. This region 
is a smooth topological annulus which we divide into $b$ pieces and on which we define $f_{1}$ to be rotation by $a / b$. At this point we continue as before, embedding a second annulus in the first and dividing this second annulus into $p_{2}$ pieces. By choosing the subsequent $p_{n}$ large we preserve the smoothness of the diffeomorphism.

Continuing with a dense set of rationals and a sequence of $\epsilon$ 's tending (rapidly) to zero, we generate a countably dense set of numbers in the rotation set for which the only corresponding minimal set would be the pseudocircle. This leads us to another question with which we conclude.

Question 2. Let $f$ be an area preserving homeomorphism of the annulus. For each irrational, $\alpha$, in the rotation set, with the exception of some countable subset, does $f$ have a minimal set $X$ so that $f \mid X$ is semiconjugate to rigid rotation by $\alpha$ on the circle?

\section{ACKNOWLEDGEMENTS}

I would like to thank G.R. Hall, P. Boyland, J. Williams, and the referee for helpful comments and suggestions.

\section{REFERENCES}

[B1] R.H. Bing, A homogeneous indecomposable plane continuum, Duke Math. J. 15 (1948), 729-742. MR 10:261a

[B2] _ Concerning hereditarily indecomposable continua, Pacific J. Math. 1 (1951), 43-51. MR 13:265b

[Bd] P. Boyland, The rotation set as a dynamical invariant, in: Twist Mappings and Their Applications, IMA Volumes in Mathematics, 44 ed. R. McGehee and K. Meyer (Springer, Berlin, 1992, pp. 73-86). MR 94i:58104

[D] R.L. Devaney, An Introduction to Chaotic Dynamical Systems, (Benjamin/Cummings, 1986). MR 87e:58142

[F] L. Fearnley, The pseudo-circle is unique, Trans. Amer. Math. Soc. 149 (1970), 45-64. MR 41:6172

[H] M. Handel, A pathological area preserving $C^{\infty}$ diffeomorphism of the plane, Proc. Amer. Math. Soc. 86 (1982), 163-168. MR 84f:58040

[M] J. Moser, Lectures on Hamiltonian systems, Mem. Amer. Math. Soc. 81(1968), 1-60. MR 37:6060

[T] M. Turpin, The restricted rotation number of an example of Handel, preprint.

Department of Mathematics, University of Hartford, West Hartford, Connecticut 06117

E-mail address: mturpin@hartford.edu 